Annls Limnol. 15 (2) 1979 : 113-121.

\title{
ILLUSTRATION CHEZ AUSTROPOTAMOBIUS PALLIPES PALLIPES LE. DES EFFETS D'UN FACTEUR TROPHIQUE : LE TAUX DES PROTEINES DE " A RATION
}

\author{
par Cl. CHAISEMARTIN 2
}

\begin{abstract}
L'écrevisse pallipède recevant la ration alimentaire la plus riche en protéines se trouve dans des conditions plus favorables, particulièrement pour la popula. tion originaire des eaux eutrophes. La traduction, parmi les tests physiologiques, est une augmentation de la fréquence des mues, du niveau des protćines de l'hémolymphe et du pourcentage de la masse musculaire abdominale.

La relation positive entre niveau des protéines de l'hémolymphe et pourcentage de chair comestible montre que la protéinémie est un bon "indice" physiologique.
\end{abstract}

\section{Observations on the effects of dietary protein levels on several physiological parameters of the crayfish Austropotamobius pallipes pallipes Le.}

The crayfish fed the highest level of protein were in the best condition. Each increase in protein resulted in a better condition of the crayfish, especially for the " eutrophic-water" population. The effect is to increase noulting frequency, haemolymph protein content and percent edible meat.

The positive relation between the protein concentrations in the haemolymph and the rate of meat production that the former are a good physiological indicator.

\section{INTRODUCTION}

Chez les Astacidae, le succès dans un élevage est fonction d'une connaissance précise des nécessités des animaux, tout particulièrement des nécessités nutritionnelles. Bien que les Ecrevisses aient été conservées en captivité pendant de longues périodes, nos connaissances sur les spectres alimentaires restent réduites.

Chez l'Ecrevisse indigène à "pattes blanches », sans prétendre mâ̂triser l'alimentation, naturelle ou artificielle, nos essais pendant les périodes été-automne 1976 et 1977 révèlent la difficulté à apporter une quantité correcte de nourriture à des Ecrevisses vivant en bassins. Les écophases, en fait les principales échelles de taille, les paramètres température et teneur de l'eau en $\mathrm{O}_{2}$, conditionnent les quantités de

1. Laboratoire d'Ecologie et Biologie générale, U.E.R. des Sciences, 123, rue Albert-Thomas, 87100 Limoges. 
l'aliment à distribuer. La présentation de la nourriture sous forme de granulés secs ou de pâte humide joue un rôle important dans la préhension et dans la vitesse du transit gastro-intestinal. Malgré les liants qui évitent le délitement précoce, la perte de particules par trituration mécanique reste importante.

Dans le domaine des besoins, l'expérimentation est rendue aussi difficile. D'après Chaisemartin (1967), l'Ecrevisse est capable de puiser directement dans l'eau certains éléments, minéraux notamment, qui font défaut dans l'apport alimentaire.

Chez les Astacidae, réputés carnivores, les protéines constituent l'un des principaux facteurs alimentaires et nous nous sommes proposés de rechercher l'influence de leur taux d'incorporation sur la croissance pondérale des jeunes pendant la deuxième année de vie. Nous avons pu abaisser le pourcentage des protéines à 40 et même 20 p. 100 de la matière sèche par une introduction plus importante dans la ration de glucides facilement utilisables. Plusieurs auteurs ont étudié l'influence d'aliments composés sur la croissance de Palaemon serratus (Cuzon 1970). Les recherches de Forster et Gabbott (1971), celles de Forster et Beard (1973) constituent un progrès dans l'élaboration d'une formule alimentaire. A notre connaissance, aucune des études précédentes n'a examiné les effets du " nourrissage » sur le cycle des métabolites chez les Astacidae.

Il est particulièrement important, dans l'interprétation des données analytiques chez les Ecrevisses que le stade du cycle d'intermue soit connu. Bien établi le faible pourcentage de chair comestible chez les Ecrevisses ayant récemment mué. L'intérêt alimentaire est cependant certain puisqu'une tonne d'Ecrevisses entières apporte à peu près la même quantité de protéines qu'une tonne de bœuf sur pied. Le rendement en viande utilisable est pratiquement le même que pour les morceaux nobles du bœuf, mais avec une somme protéique sans lipides et un taux de minéraux de 50 p. 100 supérieur.

En accord avec Kracht (1973), nous retenons deux périodes dans le cycle annuel des mues : 1) la période de diecdysis pendant laquelle toutes les mues de l'année se succèdent; 2) la période d'anecdysis, toujours plus longue que la période de diecdysis. Les événements biologiques se résument par les périodes de mues, d'acquisition des caractères sexuels adultes, de la ponte et de l'éclosion. Chez les Astacidae, mue, croissance et reproduction interviennent, apparemment, à des époques séparées : un facteur endogène, ou "horloge biologique ", programmant, selon Armitage (1973), le cycle annuel des mues et des périodes de repos.

Notre objectif est de montrer qu'un certain nombre de paramètres physiologiques varient directement avec l'apport de nourriture : un régime naturel ou une alimentation artificielle isocalorique renfermant par unité de matière sèche : 40,20,10 et 0 p. 100 de caséine. 
Sont retenus comme "indicateurs" possibles de rations alimentaires adéquates: 1) la programmation des intermues et les masses humides successivement atteintes; 2) les pourcentages d'humidité tissulaire et de la chair comestible dans la queue (en p. 100 du poids humide global) ;3) les niveaux glycémiques et protéinémiques au seuil de la période de stabilité de l'intermue.

\section{1. - MATERIEL BIOLOGIQUE ET TECHNIQUES}

Un tel objectif nécessite un grand nombre d'individus (150 au départ de chacune des quatre séries trophiques) pour surmonter les variations individuelles; des Ecrevisses rendues comparables par leur poids, leur stade dans le cycle des mues. Les vingt semaines de " nourrissage " rendent les différences alimentaires plus significatives que celles attribuées à la variation individuelle.

Les écrevisses juvéniles $A$. pallipes pallipes (Le.), capturées vers la fin de leur premier automne de vie, sont installées par lots de 50, en milieu naturel, dans des bacs en fibro-ciment pourvus de caches et alimentés en eau courante. Les paramètres édaphiques sont les suivants : dureté totale : $39 \mathrm{mg} / 1$ de $\mathrm{CaCO}_{3} ; \mathrm{Ca}^{2+}: 14 \mathrm{mg} / 1 ; \mathrm{Mg}^{2+}: 3,2$ $\mathrm{mg} / 1 ; \mathrm{Na}^{+}: 12 \mathrm{mg} / 1 ; \mathrm{HCO}_{3}: 32 \mathrm{mg} / \mathrm{l} ; \mathrm{SO}_{4}{ }^{2-}: 4,8 \mathrm{mg} / 1 ; \mathrm{Cl}^{-}: 16 \mathrm{mg} / 1$; $\mathrm{pH}: 6,9 \pm 0,2 ; \mathrm{O}_{2} ; \mathrm{O}_{2}$ dissous : de 84 à 116 p. 100 de saturation à $18{ }^{\circ} \mathrm{C} ; \mathrm{CO}_{2}: 0,5 \pm 0,2 \mathrm{mg} / \mathrm{l}$. En janvier l'eau est à environ $6{ }^{\circ} \mathrm{C}$; elle atteint $21^{\circ} \mathrm{C}$ en juillet. La lumière reçue à la surface des bacs est celle répondant à un photopériodisme naturel; celle reçue par les jeune écrevisses pallipèdes, naturellement lucifuges, reste un cas d'individu. Tous les paramètres cités restent les mêmes quelle que soit la série expérimentale. Seules varient la qualité de la nourriture (proies vivantes ou aliment artificiel plus ou moins complet) et l'origine écologique des deux populations mises en stabulation : eaux courantes, soit oligo, soit eutrophiques, aussi eucalciques. Au départ de toutes les séries d'observations, les écrevisses des eaux oligotrophes ont subi cinq exuviations pour un poids humide moyen de $330 \mathrm{mg}$; celles des eaux eutrophes ont effectué six exuviations et atteint le poids frais individuel moyen de : $550 \mathrm{mg}$.

Chez l'Ecrevisse pallipède, les "spectres " alimentaires restent mal définis et varient en fonction des proies et des végétaux disponibles. La nourriture naturelle, renouvelée toutes les semanes, comprend des moules d'eau douce ouvertes et des rhizomes d'iris. La nourriture artificielle est préparée à partir d'ingrédients secs $(50$ parties en poids), intimement mélangés avec de l'eau très chaude ( 50 parties par volume), puis réfrigérés jusqu'à ce que l'agar soit solidifié. La composition centésimale des régimes, exprimée en $\mathrm{g}$ p. 100 de la matière sèche est la suivante: cellulose: 15 ; agar: 10 ; huile de foie de 
morue : 10 ; mélange minéral ${ }^{1}$ : 5 ; mélange vitaminique de CaSTELL. (1972) ; 1 ; amidon soluble: $20,40,50$ et 60 et caséine : 40,20, 10 et 0 p. 100 pour les quatre régimes essayés et fournis au niveau de subsistance de 0,5 p. 100 du poids corporel de nourriture par jour.

Le traitement statistique des données quantitatives (intervalle de confiance à 95 p. 100) porte sur vingt écrevisses prises "au hasard " dans chacune des quatre séries expérimentales et au maximum pour chacune des trois intermues observées. La dissection de l'écrevisse, le traitement des tissus et les méthodes analytiques retenues ont été précédemment décrits (Chaisemartin 1971). Les écrevisses "échantillons" sont conservées à $-15^{\circ} \mathrm{C}$ avant les analyses. Des portions d'hépatopancréas et de muscle sont séchées à $110^{\circ} \mathrm{C}$ pendant 24 heures pour la détermination des teneurs hydriques. L'abdomen est bouilli dans un liquide physiologique pour Crustacés pendant $15 \mathrm{minu}$ tes et la viande comestible est estimée, selon Stewart (1967), en pourcentage du poids humide.

L'analyse hémolymphatique du glucose circulant répond à un prélèvement direct à la pipette automatique de $50 \mu \mathrm{l}$ dont la pointe est introduite dans la membrane articulaire à la base du protopodite. Les protéines hémolymphatiques, précipitées par le réactif de Somogyi, selon le protocole de Flemister (1958), en utilisant $0,2 \mathrm{ml}$ de $(\mathrm{BaOH})_{2}$ à 8 p. 100 puis $0,2 \mathrm{ml}$ de $\mathrm{ZnSO}_{4}$ à 8 p. 100 sont centrifugées à $3500 \mathrm{t} / \mathrm{mn}$ pendant $5 \mathrm{mn}$. Le glucose vrai est analysé à partir du surnageant par la méthode de Montgomery (1957) à la glucose-oxydase. L'analyse hémolympathique des protéines globales répond à la méthode de Lowry (1951).

\section{2. - RESULTATS}

1. Effets de la stabulation et des régimes trophiques naturels et artificiels à quatre teneurs protéiniques différentes sur l'incidence des mues et les masses moyennes enregistrées aux intermues successives (Tableau I).

Une grande variation dans les régimes alimentaires entraîne des modifications dans le cycle des mues, dans le développement et la croissance des écrevisses durant le deuxième été-automne de leur vie.

Rappelons ici que la valeur pondérale d'intermue correspond à l'accroissement définitif réalisé lorsque l'entrée d'eau est terminée, que l'animal a acquis la taille invariable qu'il conservera jusqu'à la

1. Le mélange minéral comprend, en $\mathrm{mg} / \mathrm{g}$ de mélange sec: $\mathrm{CuSO}_{4}: 507 ; \mathrm{ZnSO}_{4}$ : $352 ; \mathrm{CoCl}_{2}: 105 ; \mathrm{MnSO}_{4}: 80 ; \mathrm{AlCl}_{3}: 18 ; \mathrm{KI}: 17$. 
mue suivante et que l'eau a été, en grande partie, remplacée par des tissus. Figurer dans le temps la croissance pondérale des écrevisses passe par la détermination des taux de croissance pondéraux, liés par des relations simples au taux de croissance linéaire et par celle de la fréquence des mues.

Tableau I. - Effets du niveau des protéines alimentaires sur la situation temporelle des intermues pendant la deuxième année de vie de l'écrevisse pallipède et sur les masses humides moyennes enregistrées. Les valeurs pondérales sont exprimées en mg et suivies des limites de confiance à 95 p. 100, basées sur l'erreur standard de la moyenne de 20 écrevisses vivantes.

\begin{tabular}{|c|c|c|c|c|c|c|}
\hline \multirow{3}{*}{$\begin{array}{c}\begin{array}{c}\mathrm{N}^{\circ} \text { d'ordre } \\
\text { de } \\
\text { l'intermue }\end{array} \\
\begin{array}{c}N^{\circ} 6 \\
\text { oligotrophe }\end{array}\end{array}$} & \multicolumn{2}{|c|}{$\begin{array}{cc}\text { Alimentation } & \text { naturelle } \\
\text { témoins } & \text { témoins } \\
\text { nature } & \text { élevage }\end{array}$} & $\begin{array}{l}\text { Aliment: } \\
\text { régime: } \\
40 \% \\
\text { caséine }\end{array}$ & $\begin{array}{l}\text { ation artificiell } \\
\text { régime: } \\
20 \% \\
\text { caséine }\end{array}$ & $\begin{array}{l}\text { e ; en } \% \mathrm{~d} \\
\text { régime : } \\
10 \% \\
\text { caséine }\end{array}$ & $\begin{array}{l}\text { caséine } \\
\text { régime : } \\
0 \% \\
\text { caséine }\end{array}$ \\
\hline & $24 \mathrm{mai}$ & $21 \mathrm{mai}$ & $21 \mathrm{mai}$ & 3 juin & 9 juin & $24 \mathrm{mai}$ \\
\hline & $320 \pm 18$ & $250 \pm 22$ & $385 \pm 48$ & $355 \pm 33$ & $365 \pm 62$ & $420 \pm 76$ \\
\hline \multirow{2}{*}{$\begin{array}{l}\mathrm{N}^{\circ} 7 \\
\text { eutrophe }\end{array}$} & $15 \mathrm{mai}$ & $18 \mathrm{mai}$ & $24 \mathrm{mai}$ & $24 \mathrm{mai}$ & 15 juin & 27 mai \\
\hline & $550 \pm 48$ & $540 \pm 57$ & $725 \pm 55$ & $550 \pm 72$ & $515 \pm 49$ & $640 \pm 82$ \\
\hline \multirow{2}{*}{$\begin{array}{c}\mathrm{N}^{\circ} 7 \\
\text { oligotrophe }\end{array}$} & 12 juil. & 21 juil. & 24 juil. & 18 juil. & 15 août & \\
\hline & $555 \pm 39$ & $450 \pm 46$ & $640 \pm 52$ & $890 \pm 69$ & $630 \pm 96$ & \\
\hline \multirow{2}{*}{$\begin{array}{c}\mathrm{N}^{\circ} 8 \\
\text { eutrophe }\end{array}$} & 21 juin & 3 juil. & 27 juin & 21 juil. & 3 sept. & $\ldots \ldots$ \\
\hline & $1020 \pm 87$ & $985 \pm 84$ & $1130 \pm 110$ & $900 \pm 113$ & $735 \pm 94$ & $450 \pm 128$ \\
\hline \multirow{2}{*}{$\begin{array}{c}\mathrm{N}^{\circ} 8 \\
\text { oligotrophe }\end{array}$} & 21 août & 12 sept. & 18 août & 12 sept. & & \\
\hline & $1380 \pm 142$ & $1150 \pm 220$ & $1300 \pm 266$ & & & \\
\hline \multirow{2}{*}{$\begin{array}{c}N^{\circ} 9 \\
\text { eutrophe }\end{array}$} & 27 juil. & 3 sept. & 24 août & 6 sept. & & \\
\hline & $2220 \pm 290$ & $2170 \pm 320$ & $1785 \pm 272$ & $1400 \pm 190$ & & \\
\hline \multirow[t]{2}{*}{$\mathrm{N}^{\circ} 10$} & 15 sept. & & & & & \\
\hline & $3215 \pm 516$ & & & & & \\
\hline \multicolumn{2}{|c|}{$\begin{array}{l}\text { Masse humide globale } \\
\text { à la fin du } 2^{\circ} \text { été }\end{array}$} & $\begin{array}{l}380 \\
215\end{array}$ & $\begin{array}{l}130 \\
178\end{array}$ & $\begin{array}{l}1050 \\
1400\end{array}$ & $\begin{array}{l}630 \\
735\end{array}$ & $\begin{array}{l}380 \\
450\end{array}$ \\
\hline
\end{tabular}

Trois groupes de résultats s'affirment sous les effets de la stabulation et des régimes trophiques, naturels et artificiels à 4 teneurs protéiniques différentes sur l'incidence des mues et les masses moyennes enregistrées aux intermues successives.

a) La simple mise en élevage, en milieu naturel oligotrophique, pendant le deuxième été-automne de la vie de l'écrevisse pallipède, entraîne la disparition de l'intermue de rang « 10 », systématiquement notée dans la station d'origine eutrophique. La mue supplémentaire enregistrée chez la population des eaux eutrophes répond en fait à 
un facteur écologique de terrain dominant: une température de l'eau régulièrement plus élevée, de l'ordre de $3{ }^{\circ} \mathrm{C}$. La stabulation à unc température de l'eau inférieure fait disparaître l'intermue de rang « 10 ».

b) Les trois intermues de rangs 6,7 et $8 \mathrm{chez}$ la population d'origine oligotrophique et 7,8 et 9 chez la population d'origine eutrophique sont maintenues tant que l'apport alimentaire de protéines répond au «besoin minimal » de 20 p. 100 de la ration sèche. La séquence des mues est décalée, de l'ordre de 12 à 24 jours vers l'automne chez les écrevisses soumises aux régimes à faible teneur protéinique; elle est rompue lorsque cette teneur passe à 10 ou 0 p. 100 .

c) Les jeunes écrevisses bien alimentées s'accroissent significativement plus pour chacune des exuviations. Pour le même rang d'intermue, les masses obtenues en stabulation restent inférieures aux valeurs moyennes établies sur le terrain. La population d'origine eutrophique subit le manque à gagner le plus net : la masse obtenue lors de la troisième période de stabilité de l'année en cours ne représente que 63 p. 100 de celle naturellement escomptée. Un véritable « 0 » biologique de la croissance pondérale s'affirme dès l'intermue de rang « 8 " chez l'écrevisse d'origine eutrophique assujettie au régime à 20 p. 100 de protéines.

d) Les observations préliminaires confirment bien le rôle joué par le mode de présentation de l'aliment. Lorsque la comparaison est établie sur une même base d'apport et d'ingestion de matière sèche, ou d'énergie calorique, les rations présentées sous forme de pâte humide donnent de meilleurs résultats que la nourriture compactée sous forme de granulés secs (teneur en eau de 12,8 p. 100 du poids brut).

2. Effets du taux des protéines alimentaires sur quelques paramètres physiologiques et constantes biochimiques: humidité tissulaire; pourcentage de chair comestible des muscles abdominaux; teneurs de l'hémolymphe en glucose vrai et protéines circulantes (Tableau II).

Un certain nombre de paramètres physico-biochimiques varient directement avec l'apport de nourriture.

Une baisse du taux protéinique alimentaire, de 40 à 0 p. 100, conduit globalement à une augmentation de la perte de poids et de la teneur en eau de tous les tissus étudiés et à une diminution de la chair comestible et du taux des protéines circulantes.

a) L'eau varie peu dans le muscle lorsqu'elle est exprimée en p. 100 du poids frais. L'amplitude des variations est souvent du même ordre que celle enregistrée durant les périodes de pro- et de post-ecdysis. Globalement, la teneur en eau est plus basse au stade de prémue. Au sein de chacune des deux populations testées, le phénomène se traduit par une teneur en eau plus basse lorsque le taux de mue est élevé. 
TABleau II. - Effets du régime protéinique trophique sur les pourcentages d'humidité tissulaire $(\mathrm{H}$.$) , les pourcentages de chair comestible dans les mus-$ cles abdominaux (C.), le glucose hémolymphatique (G.), en $\mu \mathrm{g} / \mathrm{ml}$ et la protéinémie, en $\mathrm{mg} / \mathrm{ml}(\mathbf{P}$.). Chaque valeur est accompagnée des limites de confiance à 95 p. 100 calculées à partir de 12 analyses.

\begin{tabular}{|c|c|c|c|c|c|c|c|}
\hline \multirow{2}{*}{$\begin{array}{l}\mathrm{N}^{\circ} \text { d'ordr } \\
\text { de } \\
\text { l'intermue }\end{array}$} & \multicolumn{3}{|c|}{ Alimentation naturelle } & \multicolumn{3}{|c|}{$\begin{array}{c}\text { Alimentation artificielle: } \\
\text { en p. } 100\end{array}$} & \multirow{2}{*}{ 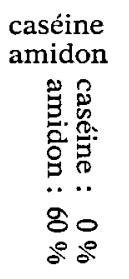 } \\
\hline & & 邕荵. & 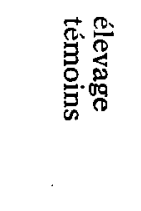 & 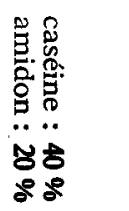 & 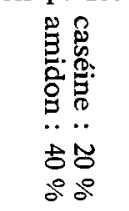 & 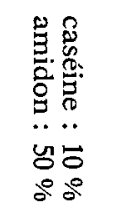 & \\
\hline \multirow{2}{*}{$\begin{array}{l}\mathrm{N}^{\circ} 6 \\
\text { oligotrophe }\end{array}$} & & 81 & 82 & 83 & 84,6 & 85 & 86,6 \\
\hline & & 18 & 15 & 15 & 11 & 8 & 7 \\
\hline \multirow{2}{*}{$\begin{array}{c}N^{\circ} 7 \\
\text { eutrophe }\end{array}$} & & 82,6 & 82,8 & 83,9 & 85,2 & 87 & 87,9 \\
\hline & & 16,4 & 14,7 & 14,2 & 10,1 & 7,2 & 5,4 \\
\hline \multirow{2}{*}{$\begin{array}{l}\mathrm{N}^{\circ} 6 \\
\text { oligotrophe }\end{array}$} & G. & $39 \pm 7$ & $41 \pm 9$ & $44 \pm 11$ & $52 \pm 17$ & $57 \pm 19$ & $64 \pm 24$ \\
\hline & P. & $32 \pm 4$ & $29 \pm 7$ & $31 \pm 6$ & $27 \pm 9$ & $25 \pm 9$ & $17 \pm 11$ \\
\hline \multirow{2}{*}{$\begin{array}{c}N^{\circ} 7 \\
\text { eutrophe }\end{array}$} & G. & $43 \pm 9$ & $45 \pm 7$ & $28 \pm 9$ & $30 \pm 14$ & $22 \pm 12$ & $16 \pm 10$ \\
\hline & P. & $27 \pm 6$ & $22 \pm 9$ & $27 \pm 6$ & $23 \pm 6$ & $18 \pm 9$ & $17 \pm 13$ \\
\hline \multirow{2}{*}{$\begin{array}{c}\mathrm{N}^{\circ} 7 \\
\text { oligotrophe }\end{array}$} & G. & $44 \pm 12$ & $47 \pm 16$ & $49 \pm 14$ & $56 \pm 14$ & $59 \pm 17$ & $66 \pm 20$ \\
\hline & P. & $36 \pm 5$ & $31 \pm 7$ & $35 \pm 11$ & $29 \pm 8$ & $24 \pm 14$ & $20 \pm 9$ \\
\hline \multirow{2}{*}{$\begin{array}{c}N^{\circ} 8 \\
\text { eutrophe }\end{array}$} & G. & $27 \pm 5$ & $29 \pm 6$ & $24 \pm 14$ & $36 \pm 9$ & $44 \pm 12$ & $49 \pm 16$ \\
\hline & P. & $33 \pm 9$ & $30 \pm 7$ & $25 \pm 10$ & $22 \pm 9$ & $19 \pm 7$ & $18 \pm 8$ \\
\hline \multirow{2}{*}{$\begin{array}{l}\mathrm{N}^{\circ} 8 \\
\text { oligotrophe }\end{array}$} & G. & $41 \pm 9$ & $43 \pm 11$ & $47 \pm 13$ & $54 \pm 14$ & $62 \pm 19$ & $67 \pm 22$ \\
\hline & P. & $32 \pm 7$ & $27 \pm 12$ & $22 \pm 12$ & $19 \pm 11$ & $16 \pm 9$ & $12 \pm 7$ \\
\hline \multirow{2}{*}{$\begin{array}{c}N^{\circ} 9 \\
\text { eutrophe }\end{array}$} & G. & $29 \pm 6$ & $34 \pm 8$ & $45 \pm 11$ & $56 \pm 17$ & $63 \pm 22$ & $69 \pm 27$ \\
\hline & P. & $34 \pm 9$ & $31 \pm 9$ & $27 \pm 7$ & $23 \pm 13$ & $14 \pm 9$ & $11 \pm 9$ \\
\hline
\end{tabular}

Dans l'hépatopancréas, une teneur en eau supérieure à 70 p. 100 répond à un stade " hors mue ».

b) L'hémolymphe est facilement pourvue à l'aide de glucose métabolisé dans le tractus digestif. Le niveau du glucose circulant est fonction de la teneur en amidon soluble dans le régime. Après vingt semaines d'expérience, le glucose hémolymphatique est inversement corrélé au niveau des protéines alimentaires, mais en relation directe avec la teneur de la ration en amidon.

c) Les protéines de l'hémolymphe, de l'ordre de 3,2 p. 100, diminuent régulièrement avec la durée d'action des régimes hypoprotéi- 
ques. Les écrevisses qui survivent au régime « 0 p. 100 de protéines » ont une protéinémie supérieure à 1,6 p. 100 .

\section{3. - DISCUSSION}

Une teneur protéinique élevée ( 20 à 40 p. 100 du régime sec) correspond à des conditions améliorées pour l'écrevisse. Kitabayashi et coll. (1971) observent, chez les Natantia, une croissance plus rapide et une survie meilleure à partir de régimes alimentaires dont la teneur protéinique est élevée. Kanazawa et coll. (1970) notent aussi une croissance accélérée avec 50 p. 100 ou plus de protéines de soja dans le régime. Foster et coll. (1973) montrent chez Palaemon serratus que l'accroissement de la taille est maximal pour une teneur en protéines supérieure à 70 p. 100 dans la ration.

Il existe une parenté très positive, abstraction faite des paramètres édaphiques et climatiques rendus identiques par la stabulation en un même lieu, entre l'incidence de la mue et la teneur des protéines alimentaires chez la population des eaux eutrophes. Cette population se nourrit essentiellement de Mollusques dans son milieu d'origine et nous comprenons l'effet plus accentué du niveau des protéines alimentaires chez elle. La question se pose de connaître si le contenu stomacal des individus de cette population renferme des niveaux élevés de protéases, ceci relativement aux autres types d'enzymes digestifs.

Certes, la caséine est utilisée comme protéine fondamentale dans un grand nombre d'études sur la nutrition car elle constitue une excellente source d'acides aminés essentiels ; elle semble d'autre part aisément digestible. Il est possible qu'un ou plusieurs acides aminés essentiels de la caséine jouent le rôle de facteurs limitants. Le taux de croissance pondérale serait-il plus élevé pour les régimes alimentaires renfermant de la cystine et du tryptophane?

\section{CONCLUSION}

Le cycle des mues, chez les écrevisses, s'additionne d'un facteur compliquant : celui de leur nutrition.

L'écrevisse pallipède, recevant la ration alimentaire la plus riche en protéines, se trouve dans des conditions plus favorables, particulièrement pour la population originaire des eaux eutrophes. La traduction, parmi les tests physiologiques, est une augmentation de la fréquence des mues, du niveau des protéines circulantes et du pourcentage de chair comestible. 
A l'action directe, celle de la masse de nourriture utilisable, s'ajoute un effet indirect affectant le phénomène de la mue et de la croissance, la résorption de l'hépatopancréas et la mobilisation des réserves gonadiques.

La relation positive entre niveau des protéines de l'hémolymphe et pourcentage des muscles abdominaux, montre que l'expression numérique du taux des protéines circulantes est un bon "indice " physiologique.

\section{TRAVAUX CITÉS}

Armitage (K. B.), Buikema (A. L.) et Willems (N. J.). 1973. - The effect of photoperiod on organic constituents and molting of the crayfish Orconectes nais. Comp. Biochem. Physiol., 44A : 431-456.

Castell (J. D.). 1972. - Essential fatty acids in the diet of rainbow trout (Salmo gairdneri) : growth, feed convension and some gross dificiency symptoms. J. Nutr., 102: 77-85.

Chaisemartin (Cl.). 1967. - Contribution à l'étude de l'économie calcique chez les Astacidae. Influence du milieu de vie. Thèse d'Etat, Poitiers, $n^{\circ}$ C.N.R.S. A.O. 1220,180 pages.

Chaisemartin ( $\mathrm{Cl}$.). 1971. - Mobilisation des réserves de métabolites chez les Astacidae: influences comparées de la stabulation à jeun et de certains états pathologiques. C. R. Soc. Biol., $165:$ 671-676.

CuzoN (G.). 1970. - Elevage et alimentation artificielle de Crangon crangon, Palaemon serratus et Penaeus kerathurus. Thèse de Doct. de Spéc. Océan. Biol., Univ. Aix-Marseille, 107 pages.

Flemister (L. J.). 1958. - Biol. Bull., $115:$ 180-200.

FORSTER (J. R. M.). 1971. - The assimilation of nutrients from compounded diets by the prawns Palaemon serratus et Pandalus platyceros. J. mar. Biol. Assoc. U. K., 51 : 943-961.

Forster (J. R. M.) et BEARD (T. W.). 1973. - Growth experiments with the prawn Palaemon serratus Pennant fed with fresh and compounded foods. Fish. Invest., $27: 1-16$.

Kanazawa (A.), Shimaya (M.), Kawasaki (M.) et Kashiwada (K.). 1970. - Nutritional requirement of prawn. I. Feeding on artificial diets. Bull. Jap. Soc. Sci. Fish., $36:$ 949-954.

Kitabayashi (K.), Shudo (K.), Nakamara (K.) et Ishawa (S.). 1971. - Studies of formula feed for Kuruma prawn. V. On the growth-promoting effects of protein level in a diet and re-examination of ingredients used. Bull. TOKAI Reg. Fish. Res. Lab. Tokyo No., 65 : 139-147.

KRATch (D.). 1973. - Influence de la température sur le cycle annuel de mue et sur les caractères sexuels externes de l'écrevisse Orconectes limosus (Rafinesque) juvénile. C. $R$. Acad. Sc., $277:$ 2777-2780.

Lowry (O. H.), Rosebrough (N. J.), Farr (A. L.) et Randall (R. J.). 1951. - Protein measurement with the Folin phenol reagent. J. Biol. Chem., $193: 265-275$.

Montgomery (R.). 1957. - Determination of glycogen. Arch. Biochem. Biophys., $67: 378-386$.

Stewart (J. E.), Cornick (J. W.), Foley (M. F.), Li (D. M.) et Bishop (C. M.). 1967. Muscle weight relationship to serum protein, hemocytes and hepatopancreas in the lobster (Homarus americanus). J. Fish. Res. Board Can., 24 : 2339-2354. 